\title{
AMALGAMATIONS OF CATEGORIES
}

\author{
JOHN MACDONALD AND LAURA SCULL
}

\begin{abstract}
We consider the pushout of embedding functors in Cat, the category of small categories. We show that if the embedding functors satisfy a 3 -for- 2 property, then the induced functors to the pushout category are also embeddings. The result follows from the connectedness of certain associated slice categories. The condition is motivated by a similar result for maps of semigroups. We show that our theorem can be applied to groupoids and to inclusions of full subcategories. We also give an example to show that the theorem does not hold when the property only holds for one of the inclusion functors, or when it is weakened to a one-sided condition.
\end{abstract}

\section{INTRODUCTION}

Taking the pushout of two objects along a common subobject is a construction which is useful in many contexts, from geometric gluing constructions of topological spaces to algebraic free products with amalgamation of groups. These can have strikingly different behaviours in different settings.

The property we are interested in is one originally observed in groups by Schreier [8], who proved that the original groups embed into their free product with amalgamation. For various kinds of algebras, conditions have been given which ensure that a Schreier embedding theorem holds $[3,4,5]$. The topic has also been studied via amalgamations: an amalgamation in a category is a pushout diagram in which all morphisms are monic (Tholen, [9]). A category is said to have the amalgamation property if amalgamations always exist for any diagram of monic maps $B \hookleftarrow A \hookrightarrow C$; such diagrams give a Schreier-type embedding result for the category in question. An extensive survey of amalgamations and related issues, including information on which categories satisfy the amalgamation property, is found in [2].

This paper considers the category of small categories, with morphisms given by functors; we denote this by Cat. This is a very general context which extends many of the usual algebraic categories to a 'many object' setting. For example, groupoids are the many object version of groups, and the amalgamated free product of groupoids comes up when considering a Van Kampen theorem for the fundamental groupoid on many basepoints of a space [1]. Cat does not in general satisfy the amalgamation property; this was shown by Trnková [10]. However, the same paper showed that if the functors are full embeddings, then the pushout will be an amalgamation.

This paper develops a more general sufficient condition for this to occur: the embedding functors must satisfy a '3-for-2' property, which we were motivated to use by conditions developed by Howie for maps of semigroups [5]. As an application, we observe that this condition holds for a pushout of groupoids along a common

1991 Mathematics Subject Classification. Primary 18A30; Secondary 18B40, 20L17.

Key words and phrases. category, pushout, amalgamation. 
subgroupoid, and so there is an embedding of the original groupoids into the free product with amalgamation groupoid. Similarly, the property holds for inclusions of full subcategories, recovering Trnková's original result. We also give an example in which the functors to the pushout are not injective, to show that the result does not hold when the property holds for only one of the inclusion functors, or when it is weakened to a one-sided condition.

The organization of the paper is as follows. Section 2 is a general discussion about pushouts of categories. Section 3 contains an explanation of the 3 -for-2 property, and the statement and proof of the main theorem. Section 4 has the applications and the example showing that the property cannot be easily weakened. Section 5 contains the proof of the key but technical lemma about connected slice categories used in the proof of the main theorem.

The authors would like to thank the referee for his or her helpful suggestions, particularly suggesting the homotopy formulation of the condition used in the main theorem, and pointing out inconsistancies in notation.

\section{Pushouts of Categories}

In this section, we give a quick overview of the general structure of a pushout in Cat.

Given any functors $F_{X}: \mathcal{W} \rightarrow \mathcal{X}$ and $F_{Y}: \mathcal{W} \rightarrow \mathcal{Y}$, we can form the pushout category $\mathcal{Z}$ which fills in the commutative diagram of functors

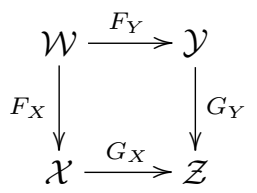

and is universal among such categories.

We will be interested in the case when the functors $F_{X}$ and $F_{Y}$ are embeddings, that is, are injective on objects and morphisms. To simplify notation, therefore, we consider $\mathcal{W}$ as a subcategory of both $\mathcal{X}$ and $\mathcal{Y}$. To create the pushout category $\mathcal{Z}$, one strategy is to start with

$$
\overline{\mathcal{Z}}=(\mathcal{Y}-\mathcal{W}) \cup(\mathcal{X}-\mathcal{W}) \cup \mathcal{W}
$$

considered as a disjoint union of objects and morphisms. Note that $\overline{\mathcal{Z}}$ is not in general a category, since even if the source and target match, the composition is not defined for $g \in \mathcal{Y}-\mathcal{W}$ and $f \in \mathcal{X}-\mathcal{W}$. However, $\overline{\mathcal{Z}}$ does have a partially defined binary operation on its morphisms $\circ$, where $f \circ g$ is defined and equal to its composition in $\mathcal{X}$ (or $\mathcal{Y}$ ) if $f$ and $g$ are both in $\mathcal{X}$ (or $\mathcal{Y}$ ) and are composable there. It is clear that we have a commutative diagram

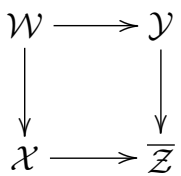

in the category of sets with partially defined binary operations.

We can adapt $\overline{\mathcal{Z}}$ to get the pushout category $\mathcal{Z}$ as follows.

- Objects of $\mathcal{Z}$ are the objects of $\overline{\mathcal{Z}}$. 
- Morphisms of $\mathcal{Z}$ are equivalence classes of finite strings of composable morphisms of $\overline{\mathcal{Z}}$.

Explicitly, a morphism is given as the equivalence class of a string $\left[\alpha_{1}, \alpha_{2}, \alpha_{3}, \ldots, \alpha_{n}\right]$ where each $\alpha_{i}$ is a morphism of $(\mathcal{Y}-\mathcal{W}),(\mathcal{X}-\mathcal{W})$ or $\mathcal{W}$ such that $\operatorname{source}\left(\alpha_{i}\right)=\operatorname{target}\left(\alpha_{i+1}\right)$ for $i=1,2, \ldots, n-1$.

When it is defined, composition is obtained by concatenation $\left[b_{1}, \ldots, b_{m}\right] \circ\left[a_{1}, \ldots, a_{n}\right]=\left[b_{1}, \ldots, b_{m}, a_{1}, \ldots, a_{n}\right]$ (note that we must have $\operatorname{source}\left(b_{m}\right)=\operatorname{target}\left(a_{1}\right)$ for this to be defined).

- The equivalences on the strings which determine the morphisms are generated by the following 'moves':

$$
\left[\alpha_{1}, \ldots, \alpha_{n}\right] \Rightarrow\left[\alpha_{1}, \ldots, \alpha_{i} \alpha_{i+1}, \ldots, \alpha_{n}\right]
$$

for each $n$-tuple where $\alpha_{i}, \alpha_{i+1}$ are both in $\mathcal{X}$ (or $\mathcal{Y}$ ) and thus their composition $\alpha_{i} \alpha_{i+1}$ is defined. Two strings of maps are equivalent if there is a finite sequence of composable generating moves connecting them, which we will refer to as a zigzag.

A description of this pushout category is given in [7].

\section{THE MAIN RESULT}

This section will use the notation from the previous section: we let $\mathcal{Z}$ denote the pushout category of functors $F_{X}: \mathcal{W} \rightarrow \mathcal{X}$ and $F_{Y}: \mathcal{W} \rightarrow \mathcal{Y}$. Again, we assume that the functors $\mathcal{W} \rightarrow \mathcal{X}$ and $\mathcal{W} \rightarrow \mathcal{Y}$ are injective on objects and morphisms, so as in the previous section, we consider $\mathcal{W}$ a subcategory of $\mathcal{X}$ and $\mathcal{Y}$. The main goal of this paper is to develop sufficient conditions under which the induced functors into the pushout category $G_{X}: \mathcal{X} \rightarrow \mathcal{Z}$ and $G_{Y}: \mathcal{Y} \rightarrow \mathcal{Z}$ are also embeddings. In the terminology of Tholen [9], this says that the pushout is an amalgamation.

Section 4 has an example which shows that this is not true for an arbitrary category; some conditions are necessary. This question of what conditions are needed has been considered by Trnková [10], who showed that when the subcategory $\mathcal{W}$ is full in both $\mathcal{X}$ and $\mathcal{Y}$, then the resulting pushout is an amalgamation.

We introduce an alternate, more general, condition on the inclusion functors which is also sufficient for the result. Our conditions are inspired by those developed by Howie [5] for an analogous result in the category of semigroups; but we express them in terms coming from homotopy theory, as in Lack [6]:

Definition 3.1. A class of morphisms $\mathcal{M}$ of $\mathcal{X}$ has the 3-for-2 property when: if $f, g$ and $h$ are morphisms in $\mathcal{M}$ with $h=g \circ f$, then if any two of $f, g$ and $h$ are in $\mathcal{M}$, the third is also in $\mathcal{M}$.

Similarly, we make the following definition.

Definition 3.2. A functor $F: \mathcal{W} \rightarrow \mathcal{Y}$ has the 3-for-2 property if the set of image morphisms $\{F(\omega) \mid \omega$ a morphism in $\mathcal{W}\}$ satisfies the 3-for-2 property.

If we have a functor $F: \mathcal{W} \rightarrow \mathcal{X}$ which is injective on objects, and $f$ and $g$ are composable morphisms in $\mathcal{X}$ which are in the image $F(\mathcal{W})$, then it is easy to see that the composition $f \circ g$ is also in the image, since any pre-images of $f$ and $g$ must be composable in $\mathcal{W}$. So for such functors, this property is really two statements: a left version which says that if $f$ is in the image of $F$ and we pre-compose with a morphism to get $f \circ g$ also in the image of $F$, then $g$ must also be in the image; and an analogous right condition about post-composition. 
These conditions are sufficient to prove our main result.

Theorem 3.3. If the functors $F_{X}: \mathcal{W} \rightarrow \mathcal{X}$ and $F_{Y}: \mathcal{W} \rightarrow \mathcal{Y}$ are embeddings which both satisfy the 3-for-2 property, then the induced functors $G_{X}: \mathcal{X} \rightarrow \mathcal{Z}$ and $G_{Y}: \mathcal{Y} \rightarrow \mathcal{Z}$ are also embeddings.

It is clear from the construction of $\mathcal{Z}$ that the functors $G_{X}$ and $G_{Y}$ are always injective on objects; it is the morphisms that need closer attention. Therefore we focus on these from here. To this end, we define the category $\mathcal{C}$ whose objects are strings of morphisms of $\overline{\mathcal{Z}}$, and whose morphisms are the generated by the 'moves' which define the equivalences to give morphisms in $\mathcal{Z}$. Explicitly, therefore, the objects of the category $\mathcal{C}$ are finite strings

$$
\left[\alpha_{1}, \alpha_{2}, \alpha_{3}, \ldots, \alpha_{n}\right]
$$

of composable morphisms of $\overline{\mathcal{Z}}$, and the morphisms are sequences of composable 'moves' on strings, defined by composing adjacent morphisms

$$
\left[\alpha_{1}, \alpha_{2}, \alpha_{3}, \ldots, \alpha_{n}\right] \Rightarrow\left[\alpha_{1}, \alpha_{2}, \alpha_{3}, \ldots, \alpha_{i} \alpha_{i+1}, \ldots, \alpha_{n}\right] \Rightarrow \ldots
$$

We saw from the description of the pushout category $\mathcal{Z}$ that the morphisms in the pushout are the connected components of this category $\mathcal{C}$. Note that the morphisms of $\overline{\mathcal{Z}}$, coming from morphisms in $\mathcal{X}$ or $\mathcal{Y}$, can be considered as a full subcategory of $\mathcal{C}$ consisting of strings of length 1 ; since the morphisms in $\mathcal{C}$ always strictly reduce the length of a string, this length 1 subcategory is discrete.

Our goal is to show that no morphisms of $\overline{\mathcal{Z}}$ are identified in the quotient category $\mathcal{Z}$; that is, no two distinct morphisms of $\overline{\mathcal{Z}}$ can be connected in the category $\mathcal{C}$. To enable us to concentrate attention on these, we make the following definitions.

Definition 3.4. An object $x$ of $\mathcal{C}$ is reduced in $\mathcal{C}$ if the only morphism in $\mathcal{C}$ with domain $x$ is the identity morphism.

Note that the length 1 strings of $\overline{\mathcal{Z}}$ are all reduced in $\mathcal{C}$

Definition 3.5. An object $z$ of $\mathcal{C}$ is reducible if there is a map from $z$ to a reduced object $x$. We say that $z$ reduces to $x$.

An object of $\mathcal{C}$ is $\overline{\mathcal{Z}}$-reducible if it reduces to a length 1 string of $\overline{\mathcal{Z}}$ (regarded as an object of $\mathcal{C})$.

Now we want to look at connected pieces of $\mathcal{C}$. In fact, we will consider the following slice categories.

For any object $x$ of $\mathcal{C}$, let $x / \mathcal{C}$ denote the full subcategory of objects under $x$ obtained by omitting the identity morphism $i d_{x}$. Therefore objects are non-identity maps $x \rightarrow c$; a morphism between two such is given by a map on the codomain making the obvious diagram commute.

The key to proving Theorem 3.3 is the following.

Proposition 3.6. Suppose $F_{X}$ and $F_{Y}$ are embedding functors which satisfy the 3-for-2 property. Then the categories $x / \mathcal{C}$ are connected for each $x$ which is $\overline{\mathcal{Z}}$ reducible.

The proof of this proposition is somewhat technical and involves looking at various cases which can arise in reducing strings. We defer the proof to Section 5 . Here, we use this proposition to prove our main result. 
Proof of Theorem 3.3. We want to show that there can be no identification of different length 1 strings of $\overline{\mathcal{Z}}$ under the equivalence relation defining $\mathcal{Z}$; that is, that no two length 1 strings can be connected in $\mathcal{C}$. To do this, we show that if $x$ is in $\overline{\mathcal{Z}}$ and $x \leftarrow z_{1} \rightarrow z_{2} \ldots$ is a zigzag of morphisms in $\mathcal{C}$, then every object $z_{i}$ in the zigzag is $\overline{\mathcal{Z}}$-reducible and reduces to the unique element $x$.

We prove this by induction on the length of the elements in the zigzag. In the base case, if all $z_{i}$ have length 1 then the only morphisms with such a $z_{i}$ as source are identities, and the statement is clear.

Now we assume that the statement holds for any zigzag of morphisms where all strings $z_{i}$ of the zigzag have length $\leq k$, and suppose that we have a zigzag $x \leftarrow z_{1} \rightarrow z_{2} \leftarrow z_{3} \ldots$ where all $z_{i}$ have length $\leq k+1$. We show that each $z_{i}$ in the zigzag is $\overline{\mathcal{Z}}$-reducible and reduces to the unique element $x$. To do this we will do a secondary induction on the length of the zigzag.

The induction starts with a length 1 zigzag $x \leftarrow z_{1}$. In this case, it is immediately clear that $z_{1}$ is $\overline{\mathcal{Z}}$-reducible, since it comes with a map to the $\overline{\mathcal{Z}}$-reducible $x$. If $z_{1}$ reduces to both $x$ and $x^{\prime}$ then we have a diagram $x \leftarrow z_{i} \rightarrow x^{\prime}$. But now $z_{1} / \mathcal{C}$ is connected by Proposition 3.6, and so there is a string of morphisms

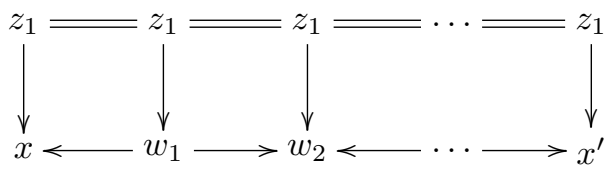

connecting the objects $z_{1} \rightarrow x$ and $z_{1} \rightarrow x^{\prime}$ in $z_{1} / \mathcal{C}$. But $z_{1}$ has length $\leq k+1$ and all non-identity morphisms of $\mathcal{C}$ strictly reduce the length of strings, so all the $w_{i}$ have length $\leq k$. So by the inductive hypothesis they are all reducible to the unique element $x$. So $x=x^{\prime}$.

Thus we have shown that the statement holds for all length 1 zigzags $x \leftarrow z_{1}$ for any $z_{1}$ of string length $\leq k+1$; and also we are assuming that it holds for all zigzags $x \leftarrow z_{1} \rightarrow z_{2} \ldots$ where $z_{i}$ has length $\leq k$ for $i \geq 2$.

Now assume inductively that the statement is true for all zigzags $x \leftarrow z_{1} \rightarrow z_{2} \ldots$. of length $\leq n$ provided each $z_{i}$ is of string length $\leq k+1$ (and also that it is true for all zigzags of arbitrary finite length provided that each $z_{i}$ is of length $\leq k$ ). Now suppose we have a zigzag $x \leftarrow z_{1} \rightarrow z_{2} \leftarrow \ldots$ of length $n+1$ with each $z_{i}$ of length $\leq k+1$. Label the $(n+1)$ st object $z_{n}$. If the last map in the zigzag is $z_{n-1} \leftarrow z_{n}$, then $z_{n}$ is reducible, since $z_{n-1}$ is; and the argument used for $z_{1}$ shows that it is uniquely reducible to $x$.

On the other hand, suppose that the last map in the zigzag goes the other way, so we have $z_{n-1} \rightarrow z_{n}$; without loss of generality this is a non-identity map. The inductive assumption ensures that $z_{n-1}$ is uniquely reducible to $x$ and we have maps $x \leftarrow z_{n-1} \rightarrow z_{n}$. Since $z_{n-1} / \mathcal{C}$ is connected by Proposition 3.6, we again get a string of morphisms

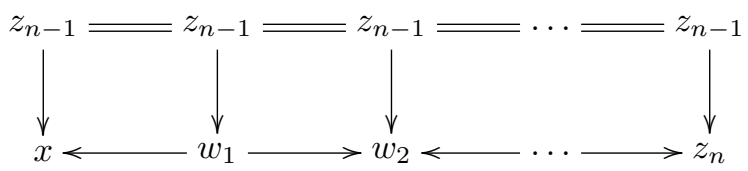

Now $z_{n-1}$ is of length $\leq k+1$, and so all the $w_{i}$ are of length $\leq k$, as is $z_{n}$ since there is a non-identity map from $z_{n-1}$. So the inductive hypothesis implies that all $w_{i}$ and $z_{n}$ reduce uniquely to $x$. 
Thus the statement is true for all zigzags of length $\leq n+1$ between strings $z_{i}$ of length $\leq k+1$. So by induction on the length of the zigzag the statement holds for all finite zigzags whose strings are of length $\leq k+1$, and then by the induction on the length of the strings, the statement holds for all finite zigzags between strings of any finite length. That is: in any zigzag, the $z_{i}$ are uniquely reducible to $x$ as desired.

Thus we have shown that in the quotient pushout category $\mathcal{Z}$, no morphisms of $\overline{\mathcal{Z}}$ are identified.

\section{Applications and Examples}

We offer some applications of the main theorem in this section, showing two situations where the 3 -for- 2 property is satisfied. We also include a counter-example where the maps into the pushout are not inclusions, which demonstrates that the condition is needed, and that several potential generalizations are not sufficient: both the left and right versions of the condition are necessary on both of the inclusion functors.

Example 4.1. A groupoid is a category in which every morphism is an isomorphism. Groupoids form a natural 'many object' generalization of groups; pushouts of groupoids occur for example in the groupoid version of the Van Kampen theorem for topological spaces with multiple basepoints. If all categories $\mathcal{X} \leftarrow \mathcal{W} \rightarrow \mathcal{Y}$ are groupoids, then the pushout of categories constructed in the previous section is also a groupoid; any morphism in $\mathcal{Z}$ is a string of morphisms from $\mathcal{X}$ and $\mathcal{Y}$, and has an inverse given by the string of inverse morphisms in the reverse order. Therefore the pushout of categories is the same as the pushout of groupoids.

It is easy to use the invertibility of the maps to show:

Lemma 4.2. If $F: \mathcal{G} \rightarrow \mathcal{H}$ is a functor between groupoids $\mathcal{G}$ and $\mathcal{H}$ which is injective on objects, then $F$ satisfies the 3-for-2 property.

Therefore by Theorem 3.3, groupoids satisfy the amalgamation property.

Example 4.3. The original situation studied by Trnková was the inclusion of full subcategories. It is easy to see that if $F: \mathcal{A} \rightarrow \mathcal{B}$ is an inclusion of a full subcategory, then $F$ satisfies the 3 -for-2 property. Thus we recover the amalgamation property for full subcategories in a simpler way via Theorem 3.3.

The next example shows that weakening the conditions of Theorem 3.3 in either of a couple of natural ways is not sufficient for the embedding result.

Example 4.4. We will consider categories $\mathcal{X} \leftarrow \mathcal{W} \rightarrow \mathcal{Y}$ where each category has the same four objects, and the functors from $\mathcal{W}$ are the obvious inclusions. 
$\mathcal{W}$

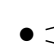
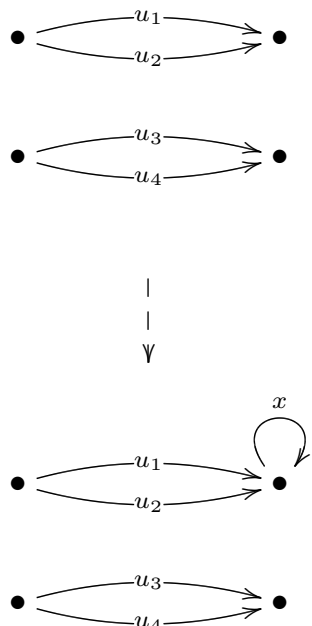

$\mathcal{X}$
$\mathcal{Y}$

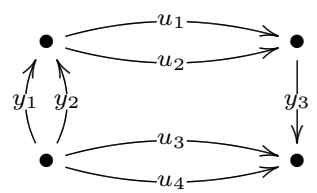

where the morphisms of $\mathcal{X}$ satisfy the relations

$$
x u_{1}=u_{1}, x u_{2}=u_{1}
$$

and the morphisms of $\mathcal{Y}$ satisfy the relations

$$
u_{1} y_{1}=u_{2} y_{2}, u_{2} y_{1}=u_{1} y_{2}, \quad y_{3} u_{1} y_{2}=u_{3}, y_{3} u_{1} y_{1}=u_{4} .
$$

In each of the original categories, $u_{3} \neq u_{4}$. However, when we form the pushout category, we can consider the following morphisms:

$$
\left[y_{3}, x, u_{1}, y_{1}\right] \sim\left[y_{3}, x u_{1}, y_{1}\right]=\left[y_{3}, u_{1}, y_{1}\right] \sim\left[y_{3}, u_{1} y_{1}\right] \sim\left[y_{3}\left(u_{1} y_{1}\right)\right]=\left[u_{4}\right]
$$

and

$$
\left[y_{3}, x, u_{2}, y_{2}\right] \sim\left[y_{3}, x u_{2}, y_{2}\right]=\left[y_{3}, u_{1}, y_{2}\right] \sim\left[y_{3}, u_{1} y_{2}\right] \sim\left[y_{3}\left(u_{1} y_{2}\right)\right]=\left[u_{3}\right]
$$

But since $u_{1} y_{1}=u_{2} y_{2}$, we also have

$$
\left[y_{3}, x, u_{1}, y_{1}\right] \sim\left[y_{3}, x, u_{1} y_{1}\right]=\left[y_{3}, x, u_{2} y_{2}\right] \sim\left[y_{3}, x, u_{2}, y_{2}\right]
$$

and so $u_{3}$ and $u_{4}$ are identified in the pushout category.

Note that in this example, the inclusion of $\mathcal{W} \rightarrow \mathcal{Y}$ satisfies the 3-for-2 property. The inclusion $\mathcal{W} \rightarrow \mathcal{X}$ does not, since $x u_{1}=u_{1} \in \mathcal{W}$ even though $x \notin \mathcal{W}$. However, it does satisfy the left version of the property: if $u x \in \mathcal{W}$ for $u \in \mathcal{W}$, then $x \in \mathcal{W}$. (Note that it would be straightforward to adapt this example to one in which the left version of the property holds but the right version fails; we would similarly get a failure of embedding into the pushout). Thus we see that a one-sided property is not sufficient, nor can we make any conclusions by having the 3 -for- 2 property on just one of the inclusion functors. 


\section{Proof of Proposition 3.6}

We give the deferred proof of the connectedness of the slice categories.

Proof. Suppose $x=\left[a_{1}, a_{2}, \ldots, a_{n}\right]$ is an $\overline{\mathcal{Z}}$-reducible string. We need to show that any two elements $x \rightarrow c$ and $x \rightarrow c^{\prime}$ of $x / \mathcal{C}$ can be connected by a finite sequence of morphisms. It is sufficient to show this in the case when both $c$ and $c^{\prime}$ come from applying one generating move to the string $x$ : any string coming from more than one move is connected to the result of the first move. Furthermore, since $x$ is $\overline{\mathcal{Z}}$-reducible there is some move $x \rightarrow c^{\prime \prime}$ with $\overline{\mathcal{Z}}$-reducible codomain; therefore it is sufficient to show that $x \rightarrow c$ and $x \rightarrow c^{\prime}$ can always be connected when one of them has $\overline{\mathcal{Z}}$-reducible codomain.

Thus we consider the following situation:

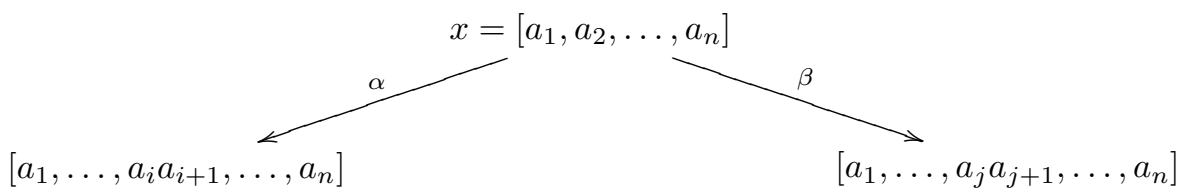

where one of $\alpha$ and $\beta$ has a $\overline{\mathcal{Z}}$-reducible codomain.

First, observe that if $i$ and $j$ differ by 2 or more, these moves involve distinct elements of the string and we can complete the diamond

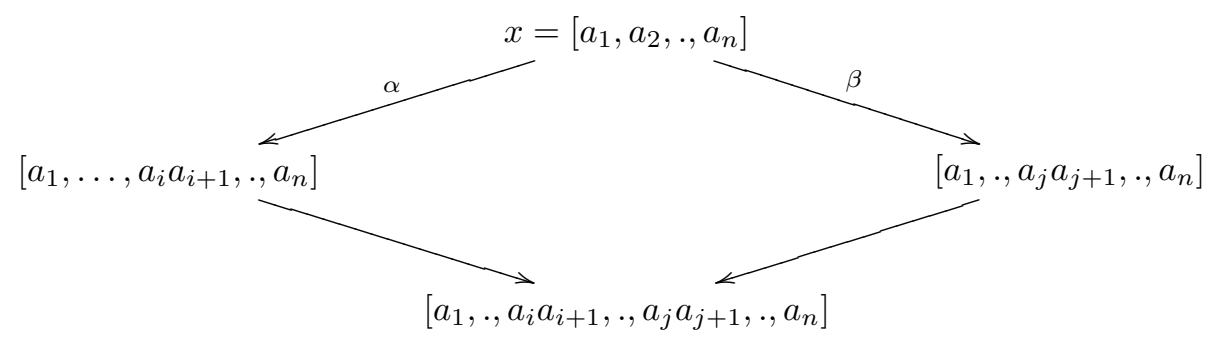

Thus $\alpha$ and $\beta$ are easily seen to be connected in $x / \mathcal{C}$.

Now suppose that $i$ and $j$ only differ by 1 ; assume without loss of generality that $i=j-1$. If $a_{i}, a_{i+1}$ and $a_{i+2}$ are all elements of $\mathcal{X}$ (or $\mathcal{Y}$ ), then the composition $a_{1} a_{i+1} a_{i+2}$ is defined; the associativity of this composition gives us the diagram

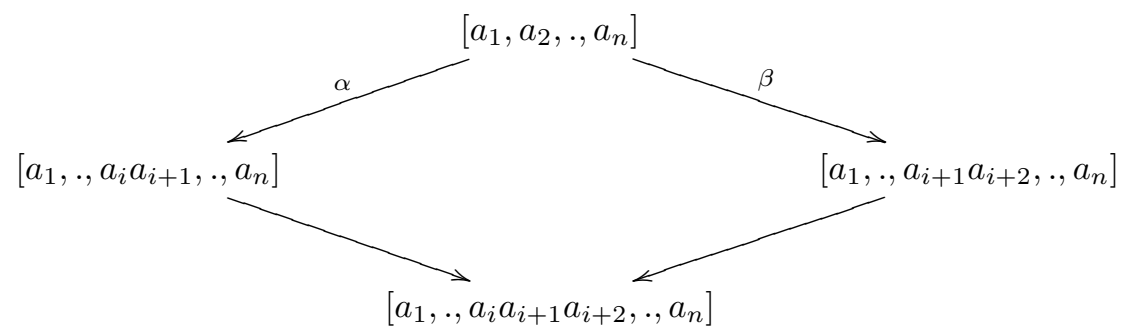

and again we see that $\alpha$ and $\beta$ are connected.

Alternately, $a_{i}, a_{i+1}$ and $a_{i+2}$ may not all be in one of $\mathcal{X}$ or $\mathcal{Y}$. Since the two moves $\alpha$ and $\beta$ are defined, if $a_{i}$ and $a_{i+2}$ are both in $\mathcal{X}-\mathcal{W}$, then all three must be in $\mathcal{X}$; similarly for $\mathcal{Y}$. So this alternate situation can only arise if $a_{i}$ is in $\mathcal{X}-\mathcal{W}$ and $a_{i+2}$ is in $\mathcal{Y}-\mathcal{W}$ (Case A) or vice versa (Case B). In these cases, in order for the moves $\alpha$ and $\beta$ to be defined, the middle element $a_{i+1}$ must be in $\mathcal{X} \cap \mathcal{Y}=\mathcal{W}$. 
Now recall that we may assume that at least one of the moves $\alpha$ and $\beta$ has $\overline{\mathcal{Z}}$-reducible codomain. First, assume that it is $\alpha$. Therefore there exists a move $\alpha^{\prime}:\left[a_{1}, \ldots, a_{i} a_{i+1}, \ldots, a_{n}\right] \rightarrow c$ for some $\overline{\mathcal{Z}}$-reducible $c$. If this move is of the form $\left[a_{1}, \ldots, a_{i} a_{i+1}, \ldots, a_{n}\right] \rightarrow\left[a_{1}, \ldots, a_{i} a_{i+1}, \ldots, a_{k} a_{k+1}, \ldots, a_{n}\right]$ for $k>i+2$, then we can connect $\alpha$ and $\beta$ as follows:

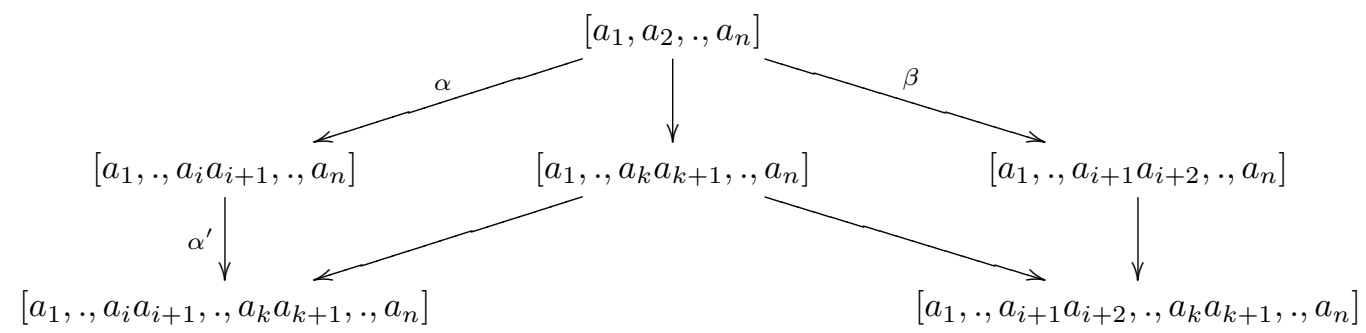

If $k<i-1$ we get a similar diagram connecting $\alpha$ and $\beta$.

Thus we have 3 remaining possibilities for the reducible move out of $\alpha$ :

(1) $k=i-1$ and $\alpha^{\prime}:\left[a_{1}, \ldots, a_{i} a_{i+1}, \ldots a_{n}\right] \rightarrow\left[a_{1}, \ldots, a_{i-1}\left(a_{i} a_{i+1}\right), \ldots, a_{n}\right]$

(2) $k=i+1$ and $\alpha^{\prime}:\left[a_{1}, \ldots, a_{i} a_{i+1}, \ldots a_{n}\right] \rightarrow\left[a_{1}, \ldots,\left(a_{i} a_{i+1}\right) a_{i+2}, \ldots, a_{n}\right]$

(3) $k=i+2$ and $\alpha^{\prime}:\left[a_{1}, \ldots, a_{i} a_{i+1}, \ldots a_{n}\right] \rightarrow\left[a_{1}, \ldots, a_{i} a_{i+1}, a_{i+2} a_{i+3}, \ldots, a_{n}\right]$

We start with scenario (2) from the list above, which we will show cannot occur under our assumptions. In this scenario, we have a diagram

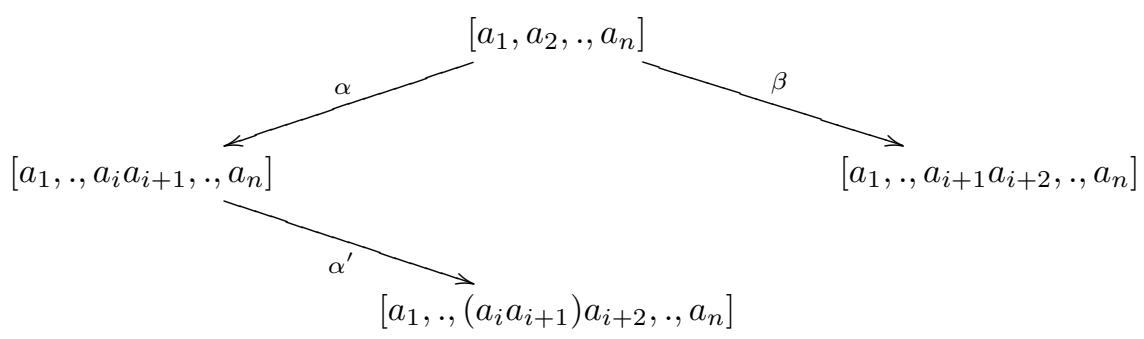

Now recall that either

(A) $a_{i}$ is in $\mathcal{X}-\mathcal{W}, a_{i+1}$ is in $\mathcal{W}$ and $a_{i+2}$ in $\mathcal{Y}-\mathcal{W}$, or else

(B) $a_{i}$ is in $\mathcal{Y}-\mathcal{W}, a_{i+1}$ in $\mathcal{W}$ and $a_{i+2}$ in $\mathcal{X}-\mathcal{W}$

Note that the composite $\left(a_{i} a_{i+1}\right) a_{i+2}$ is defined. In case $\mathrm{A}, a_{i+2} \in \mathcal{Y}-\mathcal{W}$, and so $a_{i} a_{i+1}$ must be in $\mathcal{X} \cap \mathcal{Y}=\mathcal{W}$. But this cannot happen: $a_{i}$ is in $\mathcal{X}-\mathcal{W}$ and the inclusion $\mathcal{W} \rightarrow \mathcal{X}$ satisfies the 3 -for-2 property (actually, we only need the right version here), so composing with the element $a_{i+1}$ of $\mathcal{W}$ cannot land in $\mathcal{W}$. Similarly in case B, $a_{i+2} \in \mathcal{X}-\mathcal{W}$, and so $a_{i} a_{i+1}$ must be in $\mathcal{X} \cap \mathcal{Y}$, and hence in $\mathcal{W}$. But again this is impossible: $a_{i}$ is in $\mathcal{Y}-\mathcal{W}$ and the inclusion $\mathcal{W} \rightarrow \mathcal{Y}$ also satisfies the (right) 3 -for-2 property, so $a_{i} a_{i+1}$ cannot land in $\mathcal{W}$. Therefore scenario (2) is impossible.

Next, we look at scenario (3). In Case A, the fact that $a_{i+2} a_{i+3}$ is defined means that $a_{i+3}$ must be in $\mathcal{Y}$; therefore $a_{i+1}, a_{i+2}, a_{i+3}$ are all in $\mathcal{Y}$, and can be composed. Similarly in Case B, the fact that $a_{i+2} a_{i+3}$ is defined means that $a_{i+3}$ must be in $\mathcal{X}$; here $a_{i+1}, a_{i+2}, a_{i+3}$ are all in $\mathcal{X}$. In either case, $\alpha$ and $\beta$ can be connected as 
shown in the following diagram:

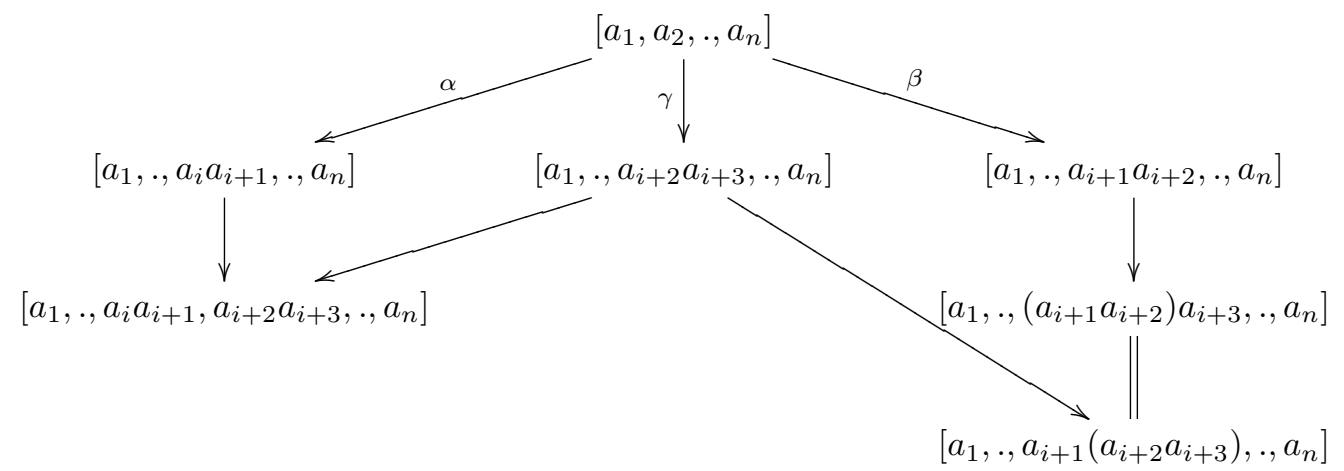

Lastly, we consider scenario (1). Here, we observe that the composite $a_{i-1}\left(a_{i} a_{i+1}\right)$ must be defined. In Case $\mathrm{A}, a_{i}$ is in $\mathcal{X}-\mathcal{W}$, and so $a_{i} a_{i+1}$ must also be in $\mathcal{X}-\mathcal{W}$, using the 3 -for- 2 property (again, the right version) of the inclusion of $\mathcal{W}$. Therefore, for the product $a_{i-1}\left(a_{i} a_{i+1}\right)$ to be defined, we must have that $a_{i-1}$ is in $\mathcal{X}$, and so all of $a_{i-1}, a_{i}, a_{i+1}$ are in $\mathcal{X}$. Similarly in Case B, the (right) 3 -for-2 property on the inclusion $\mathcal{W} \rightarrow \mathcal{Y}$ implies that $a_{i} a_{i+1} \in \mathcal{Y}-\mathcal{W}$ and cannot be in $\mathcal{W}$. Thus $a_{i-1}$ must be in $\mathcal{Y}$ for the composite $a_{i-1}\left(a_{i} a_{i+1}\right)$ to be defined; and so $a_{i-1}, a_{i}$ and $a_{i+1}$ are all in $\mathcal{Y}$.

In either case $\mathrm{A}$ or $\mathrm{B}$, we can define the following diagram connecting $\alpha$ and $\beta$.

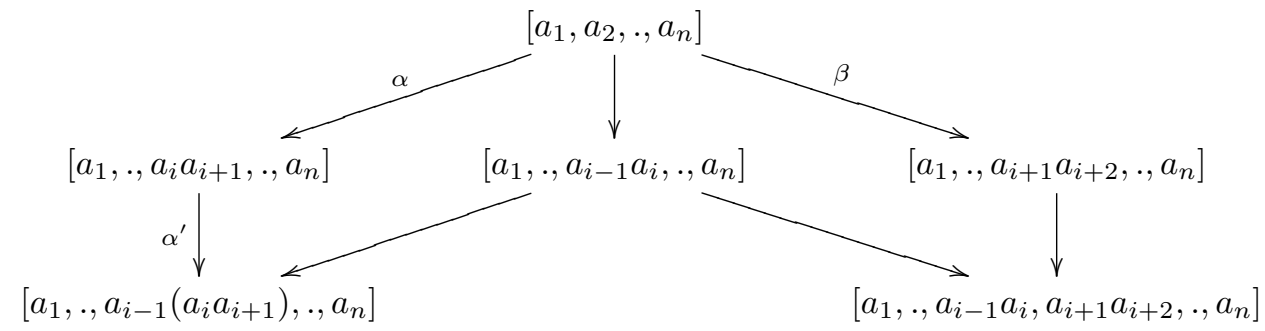

We briefly consider the symmetric cases where $\beta$ is $\overline{\mathcal{Z}}$-reducible. If the $\overline{\mathcal{Z}}$ reducible move $\beta^{\prime}:\left[a_{1}, \ldots, a_{i+1} a_{i+2}, \ldots, a_{n}\right] \rightarrow\left[a_{1}, \ldots, a_{i+1} a_{i+2}, \ldots, a_{k} a_{k+1}, \ldots a_{n}\right]$ has $k>i+2$ or $k<i-1$, we can connect $\alpha$ and $\beta$ easily by completing a diamond as above. We also have the following special cases to consider:

(3) $k=i-1$ and $\beta^{\prime}:\left[a_{1}, \ldots, a_{i+1} a_{i+2}, \ldots a_{n}\right] \rightarrow\left[a_{1}, \ldots, a_{i-1} a_{i}, a_{i+1} a_{i+2}, \ldots, a_{n}\right]$

(2) $k=i$ and $\beta^{\prime}:\left[a_{1}, \ldots, a_{i+1} a_{i+2}, \ldots a_{n}\right] \rightarrow\left[a_{1}, \ldots, a_{i}\left(a_{i+1} a_{i+2}\right), \ldots, a_{n}\right]$

(1) $k=i+2$ and $\beta^{\prime}:\left[a_{1}, \ldots, a_{i+1} a_{i+2}, \ldots a_{n}\right] \rightarrow\left[a_{1}, \ldots,\left(a_{i+1} a_{i+2}\right) a_{i+3}, \ldots, a_{n}\right]$

These cases are dealt with in a similar manner to their corresponding cases above. The case $k=i$ can be shown not to occur using the left version of the 3 -for-2 properties of the inclusions of $\mathcal{W}$ in $\mathcal{X}$ and $\mathcal{Y}$, analogous with scenario (2) from above. The case $k=i-1$ leads to a diagram connecting $\alpha$ and $\beta$ through $\left[a_{1}, \ldots, a_{i-1} a_{i}, \ldots, a_{n}\right]$ as in Scenario (3) above. And in the case $k=i+2$, again left version of the 3 -for- 2 properties can be used to show that we can define connections analogous to those of Scenario (1) above. 


\section{REFERENCES}

[1] R. Brown, 'Topology: a geometric account of general topology, homotopy types and the fundamental groupoid', John Wiley \& Sons (1988).

[2] E. W. Kiss, L. Márki, P. Pröhle and W. Tholen, 'Categorical algebraic properties. A compendium on amalgamation, congruence extension, epimorphisms, residual smallness, and injectivity', Studia Scientiarum Mathematicarum Hungarica vol 18 pp 79 - 141 (1983).

[3] J. MacDonald, 'Embeddings of Algebras', Fields Institute Communications vol 43 pp 359-373 (2004).

[4] J. MacDonald, 'Coherence and Embeddings of Algebras', Math. Z. vol 135 pp 185-220 (1974).

[5] J. Howie, 'Fundamentals of Semigroup Theory', London Mathematical Society Monographs New Series 12 (1995).

[6] S. Lack, 'A Quillen Model Structure of 2-Categories', K-Theory vol 126 pp 171-205 (2002).

[7] H. Schubert, Categories, Springer-Verlag (1972).

[8] O. Schreier, 'Die Untergruppen der freien Gruppen', Abh. Math. Sem. Univ. Hamburg vol 5 pp $161-183$ (1927).

[9] W. Tholen, 'Amalgamations in categories', Algebra Universalis vol 14 pp 391-197 (1982) .

[10] V. Trnková, 'Sums of categories with amalgamated subcategory', Comment. Math. Univ. Carolinae vol 6 pp 449-474 (1965).

The University of British Columbia, Department of Mathematics, 1984 Mathematics Road, VANCOUVER, BC, CANADA

E-mail address: johnm@math.ubc.ca, scull@math.ubc.ca 